\title{
La moral kantiana en la década del silencio. Elementos para una reconstrucción*
}

\author{
José SANTOS HERCEG \\ Departamento de Filosofía y Humanidades \\ Universidad Alberto Hurtado \\ jsantos@uahurtado.cl
}

\begin{abstract}
Resumen: El presente artículo es un intento por rastrear en las Lecciones y los Manuscritos sobre filosofía moral de Kant algunos elementos para una reconstrucción de lo que podría ser una teoría moral durante la llamada "década del silencio".

Palabras clave: motivación, debilidad, bien supremo, Dios
\end{abstract}

\begin{abstract}
This paper intends to serch in Kant's Lectures and Manuscripts on Moral Philosophy some elements that make posible a reconstruction of that what may be a Moral Theory during the "silent Decade".

Key words: motivation, weakness, highest good, God
\end{abstract}

Con la Dissertatio de 1770 se produce lo que se ha considerado el "giro más significativo" del pensamiento moral de Kant. ${ }^{1}$ Como es bien sabido, éste no es un escrito sobre moral; sin embargo, hay allí dos frases, escritas tal vez al pasar, que dan cuenta inequívoca de un cambio fundamental de su pensamiento en estas materias. ${ }^{2}$ Hasta entonces Kant sostuvo una suerte de "moral del sentimiento". En varios textos de esta etapa se encuentran pasajes en los que el autor expresamente pone las bases de una "Gefühlsmoral". ${ }^{3}$ Es en la Dissertatio donde establece, por primera vez, el papel esencial que tendrá la "razón pura" para su concepción de la moral, en cuanto que será ella la llamada a elevar el juicio de moralidad. Con un par de alusiones algo descontextualizadas, Kant se aleja, sin explicación ni desarrollo alguno, del sentimentalismo que había marcado su periodo precrítico y fija su atención en la razón pura como el elemento determinante de la moral. ${ }^{4}$ Luego de la Dissertatio, sin embargo, sobreviene una

* El texto base para la elaboración de este artículo lo constituye una conferencia presentada en el congreso "Kant a los 200 años de su muerte (12/2/2004)", organizado por la Pontificia Universidad Católica de Chile, Santiago de Chile, 25 al 27 de mayo de 2004.

${ }^{1}$ De esta opinión son, por ejemplo, Foerster (Der Entwicklungsgang der kantischen Ethik bis zur Kritik der reinen Vernunft, p. 30), P. Menzer ("Der Entwicklungsgang der Kantischen Ethik in den Jahren 1760 bis 1785", p. 51), y O. Thon (Die Grundpinzipien der Kantischen Moralphilosphie in ihrer Entwicklung, pp. 6-7).

${ }^{2}$ Cfr. KGS.II.395 y 396.

3 "En nuestros días se ha comenzado a captar que la capacidad para representar la verdad es el entendimiento, pero la capacidad para percibir lo bueno es el sentimiento, y que ambas capacidades no deben ser confundidas" ("Nachrichten über der Einrichtung seiner Vorlesungen", KGS.II.299; véase también KGS.II.217 y 311).

${ }^{4}$ Sobre este giro se ha escrito bastante. Por referirnos sólo a los textos clásicos sobre el tema, véanse, por ejemplo: P.A. Schilpp, Kant's Pre-Critical Ethics (pp. 89-106); J. Schmucker, 
"década de silencio": Kant no da a la luz pública escrito alguno que sirva para entrever lo que ocurre con sus concepciones, y habrá que esperar hasta la aparición de su gran obra, la Crítica de la razón pura (1781), para volver a leer algo de él. Si el interés es el tema moral, la espera es aún mayor, pues la Fundamentación de la metafísica de las costumbres, su primera obra sobre moral, se publica recién en 1785 . Lo que haya pasado en estos quince años, desde la insinuación de un cambio radical de perspectiva, hasta la confección, articulación y publicación de toda su teoría moral, no está, en principio, documentado. De hecho, en la literatura hay muy poco escrito sobre esta fase. El siguiente es un esfuerzo por paliar en parte esta deficiencia, esto es, por aportar algunos elementos que contribuyan a ir reconstruyendo inicialmente el pensamiento moral kantiano durante esta etapa.

\section{Lecciones y manuscritos}

Las únicas fuentes que pueden dar cuenta de este vacío son aquellas Lecciones (Vorlesungen) que Kant dictara en esta época y la porción del Legado manuscrito (Schriftliche Nachlaß) en la que se trata el tema moral. La publicación tanto de las primeras como del segundo era, como se sabe, parte del plan original que W. Dilthey ideó en 1899 para la publicación de la edición crítica de las Obras completas de Kant. Erich Adickes fue quien asumió la tarea de juntar, interpretar, ordenar, organizar y publicar el material de los Manuscritos; sin embargo, murió antes de finalizar su labor y Friedrich Berger prosiguió el trabajo de su maestro, hasta que en 1934 logra hacer la publicación, en el tomo XIX, de la obra crítica. ${ }^{5}$ Las Lecciones sobre filosofía moral, por su parte, lograron ser publicadas recién entre 1974 y 1979, y fue Gerhard Lehmann quien lo hizo posible. La sección IV, en particular el tomo XXVII de las Obras completas de la Academia, corresponde a las Lecciones de Kant sobre el tema moral. ${ }^{6}$ Es necesario destacar, no obstante, que algunas de las Lecciones sobre filosofía moral ya habían

Die Ursprünge der Ethik Kants in seinen vorkritischen Schriften und Reflexionen (pp. 259-277), y K. Ward, The Development of Kant's View of Ethics (pp. 42-50).

${ }^{5}$ Del Legado manuscrito ha aparecido recientemente una traducción de aquellas anotaciones agrupadas bajo el título Reflexionen zur Moralphilosophie y que corresponde al tomo XIX de la edición de la Academia, entre las pp. 91 y 317 (Reflexiones sobre filosofía moral, trad. José Santos Herceg, Sígueme, Salamanca, 2004).

${ }^{6}$ Dicho tomo consta de tres volúmenes. En el primero de ellos están los manuscritos: "Praktische Philosophie Herder" (1764-1765), "Praktische Philosophie Powalski" (1777), y "Moralphilosophie Collins" (1784-1785). El segundo volumen recoge la "Metaphysik der Sitten Vigilantius" (1793-1794) y la Ethica philosophica de Baumgarten. Finalmente, el tercer volumen contiene estudios de Lehmann y un aparato crítico junto con la "Naturrecht Feyerabend" (1784) y la "Moral Mrongovius" (1782). Sólo existe una traducción de la "Moralphilosophie Collins", hecha por Roberto Rodríguez Aramayo y Concha Roldán Panadero (Lecciones de ética, Crítica, Barcelona, 1988). 
sido publicadas cincuenta años antes por P. Menzer fuera de la edición crítica. $^{7}$

Tanto las Lecciones como los Manuscritos sobre filosofía moral están, entonces, al alcance de quien hoy se interesa por la evolución del pensamiento moral del Kant, especialmente en lo que se refiere al "periodo de silencio". Pese a ello, estos escritos han tenido una escasa repercusión; de hecho, el libro de Keith Ward de 1972, The Development of Kant's View of Ethics, ha sido el último comentario completo publicado sobre la evolución del pensamiento moral de Kant. ${ }^{8}$ Los textos de referencia sobre el tema siguen siendo el libro de Paul Schilpp, Kant's Pre-Critical Ethics, publicado en 1938, y el estudio de Josef Schmucker, Die Ursprünge der Ethik Kants in seinen vorkritischen Schriften und Reflexionen, editado en 1961. La aparición de las Lecciones no ha provocado a la crítica en ningún sentido, como podría haberse esperado. Los Manuscritos, por su parte, han sido objeto de algunos estudios; pero, al igual que las Lecciones, han caído en el olvido. ${ }^{9}$

La causa de esta falta de interés y de que hasta hoy estos textos hayan sido ignorados se encuentra en el hecho de que es posible dudar que efectivamente puedan ser fuentes fidedignas del pensamiento de Kant. Se pone en cuestión su autenticidad y su confiabilidad; ésta es la razón por la cual los editores de dos de las principales obras de Kant que existían antes de la publicación de la obra crítica se negaron expresamente a incluirlos. ${ }^{10} \mathrm{El}$ mismo Lehmann era consciente de este problema respecto de las Lecciones y lo hace ver en su introducción. ${ }^{11} \mathrm{El}$ origen de esta duda se encuentra en las peculiaridades de estos textos que los hacen poco dignos de confianza. De hecho, sin ir más lejos, en ambos casos se trata de escritos que no fueron pensados por Kant para su publicación.

Lo que se ha llamado Legado manuscrito constituye un material sumamente heterogéneo. En esta categoría se incluyen desde las notas escritas

${ }^{7}$ Con ocasión de los 200 años del nacimiento de Kant, Paul Menzer edita el libro Immanuel Kant. Eine Vorlesung über Ethik. Este texto es el resultado de la fusión de tres manuscritos diferentes: "Philosophie practica universalis" de F. Bauer (1780), las "Vorlesungen über die Philosophische Moral" de G. Kutzner (1781) y la "philosophische Moral" de Mrongovius (1782).

${ }^{8}$ Sobre bibliografía más reciente acerca del tema se pueden referir los trabajos de Lehmann, quien como editor de las Lecciones, tiene una serie de escritos sobre ellas. Se puede mencionar también el trabajo de Ada Lamacchia, de Rodríguez Aramayo y, sobre todo, el segundo capítulo del libro de Hermann Schmitz Was wollte Kant? (véase la bibliografía).

${ }^{9}$ Acerca del Legado manuscrito, cabe mencionar que tanto Schilpp como Schmucker se hacen cargo de él en sus respectivos libros. Schilpp incluso traduce un gran número de anotaciones y Schmucker dedica todo un capítulo a su estudio. Posteriormente sólo se pueden mencionar los estudios de Forschner.

${ }^{10}$ Cfr. Immanuel Kant's Sämmtliche Werke, editado por Rosenkranz y Schubert, Leipzig, 1838, e Immanuel Kants Sämmtliche Werke in chronologischer Reihenfolge, editado por Hartestein, Leipzig, 1867.

${ }^{11}$ Cfr. KGS.XXIV.965. 
al pasar sobre algún pedazo de papel, pasando por los comentarios hechos en los márgenes de los compendios con los que Kant dictaba clases, hasta los apuntes inconclusos de algún libro que se publicó o que tal vez nunca llegó a publicarse. A esta heterogeneidad hay que agregar que se trata de una enorme cantidad de material sumamente fragmentario y en ocasiones totalmente descontextualizado. No son, en caso alguno, textos con un desarrollo; son sólo extractos, esquemas, esbozos, fracciones de ideas. Las relaciones entre uno y otro son, además, muy difíciles de establecer, lo que lleva al problema del orden cronológico de estos escritos. En muchos de ellos no existe ninguna marca expresa que indique la fecha en que esas notas fueron tomadas, lo que hace imposible, en principio, situarlas temporalmente. ${ }^{12}$

Las Lecciones, por su parte, son escritos que no fueron redactados por Kant mismo, sino que son los "apuntes" de clases tomados por los alumnos que asistieron a los seminarios o lecciones dictados por él. La calidad y fidelidad de dichas notas está, por lo tanto, en relación directa con el alumno que las escribió. Por lo demás, está claro que nunca pueden ser absolutamente textuales, ${ }^{13}$ sin hacer alusión al problema de que, como se sabe, a partir de 1778 todas las lecciones que se dictaran en las universidades alemanas debían hacerse de acuerdo con algún "compendio", ${ }^{14}$ por lo que en ocasiones es muy difícil distinguir en la exposición las posturas de Kant de lo señalado por el autor del texto base. Como si lo anterior fuera poco, los manuscritos que se han preservado, es decir, los que no están fragmentados, ${ }^{15}$ presentan versiones diferentes y en ocasiones hasta contradictorias sobre el mismo punto, y estas contradicciones se extienden incluso a la obra publicada por el autor.

Ésas son las características que han llevado a algunos a desconfiar de las Lecciones y de los Manuscritos como fuentes de las cuales se puedan extraer antecedentes fructíferos que iluminen la evolución del pensamiento moral de Kant. Se ha señalado con razón, en especial en el caso de las Lecciones, que se trata de textos cuya autenticidad no está demostrada, que su credibilidad puede ser puesta en duda. La utilización de estos escritos, según

\footnotetext{
${ }^{12}$ Más detalles sobre estos problemas se encuentran en José Santos Herceg, "Estudio introductorio", en I. Kant, Reflexiones sobre filosofía moral, pp. 9-28.

${ }^{13}$ Por esta razón escribe P. Menzer, refiriéndose a las Lecciones, que "ninguna palabra, ninguna formulación debe en caso alguno ser tomada como si estuviéramos frente a alguno de los propios escritos de Kant" (Menzer, op. cit., KS.3, p. 58).

${ }^{14}$ Un "Rescripto ministerial" del 16 de octubre de 1778 establece que, de acuerdo con el lema "el peor compendio es con toda seguridad mejor que ninguno", todos los profesores deberán guiarse en sus clases por sendos libros de texto con la libertad, por supuesto, de mejorarlos en aquellos puntos en que su sabiduría personal se lo permitiera. Kant no es ni puede ser una excepción a este mandato.

${ }^{15}$ La "Praktische Philosophie" de Herder, por ejemplo, comienza en la mitad de una frase y, luego de 86 páginas, simplemente se interrumpe antes de concluir una idea.
} 
esta postura, simplemente conduce a errores de interpretación, puesto que de ellos se desprende una imagen distorsionada de lo que pueda haber sido la evolución de la reflexión kantiana sobre el problema moral. Pese a todas las dificultades, hay otros intérpretes que han insistido en su riqueza y su necesaria utilización. Ésta es justamente la razón que lleva a Dilthey a considerar su publicación en la obra crítica. ${ }^{16}$ También Erich Adickes tenía clara la importancia del material que estos escritos proporcionan. Las Reflexiones hacen posible, dice, "echar una mirada a lo profundo del taller del espíritu [de Kant], al germen y al desarrollo de su pensamiento". ${ }^{17}$ De la misma opinión son autores como G. Gerhardt ${ }^{18}$ y G. Lehmann, ${ }^{19}$ quienes han sido editores de las Lecciones. ${ }^{20}$

La confianza en estas fuentes, por supuesto, no es ciega. No deben olvidarse sus deficiencias, aunque tampoco hay que extremar las conclusiones e invalidarlas como material. Está claro que no son textos que puedan ser comparados con los escritos publicados por el propio Kant. Esto no significa, sin embargo, que no se puedan emplear; simplemente implica que, para hacerlo, se debe ser especialmente cuidadoso. ${ }^{21}$ Sin duda hay que tomar algunas medidas si se trabaja con estas fuentes, con lo que estos escritos se transforman en una cantera interesante y rica para analizar la evolución del pensamiento moral kantiano.

\section{Diiudicatio $y$ executio}

Según los textos de las Lecciones sobre filosofía moral y del Legado manuscrito, hay en principio dos problemas que deben ser resueltos cuando se piensa el tema moral: el primero de ellos se refiere a la manera en que se distingue aquello que es moralmente correcto de lo que no lo es, es decir, la forma en que se determina el bien moral y se lo separa de lo moralmente reprochable. A esto lo llamará Kant en estos escritos "diiudicatio", expresión proveniente del latín y que, como es bien sabido, significa "juicio". La diiudicatio tiene que ver con la pregunta "¿qué es lo moralmente bueno?" El segundo problema viene aparejado con el

\footnotetext{
${ }^{16}$ De acuerdo con sus propias palabras (en el prólogo a las Obras completas), estos escritos permitirían obtener una entrada a la historia de la evolución de Kant (KGS.I.XIV).

${ }^{17}$ Erich Adickes, "Introducción a la sección del Legado Manuscrito", KGS.XXIV.

${ }^{18}$ Cfr. Gerhardt, "Zur Neuausgabe", en I. Kant, Eine Vorlesung über Ethik, p. 292.

${ }^{19}$ Cfr. KGS.XXIV.965.

${ }^{20}$ Sobre este tema, cfr. José Santos Herceg, "Reivindicación de las Lecciones y Reflexiones como fuentes de la filosofía moral de Kant", pp. 555-570.

${ }^{21}$ Una primera precaución, tal vez la principal, sería trabajar con todos los manuscritos de las Lecciones en conjunto y cotejarlos permanentemente con las doctrinas del Legado manuscrito; de esta forma se logra establecer, con un alto grado de probabilidad, la autenticidad de muchas de las doctrinas allí expuestas. Ésta es la conclusión a la que han llegado tanto P. Menzer (op. cit., p. 59) como E. Arnold (KGS.V, Berlín, 1909) y Schmucker (op. cit., p. 311).
} 
anterior y se refiere a la realización efectiva de aquello que ha sido considerado bueno y la real abstención de hacer eso que se juzgó incorrecto. La palabra en castellano para "executio", que es como llama Kant a este asunto, es "ejecución" en su sentido de "llevar a cabo". La pregunta que está detrás de este problema es "¿qué me mueve a actuar de acuerdo con esa ley?"22

Lo primero, entonces, es determinar si algo es moralmente aceptable. A partir de los esbozos fijados en la Dissertatio de 1770 queda establecido que todo lo relacionado con la diiudicatio tendrá que ver con la razón pura. Esta idea permanece vigente después. ${ }^{23}$ "El juzgar — dice en las Leccioneses una acción del entendimiento acerca de ciertos actos." 24 El juicio de moralidad es de competencia exclusiva de la razón, será ella y sólo ella la que se pronuncie sobre aquello que en las Lecciones y Manuscritos se llama la bonitaet de una acción. El concepto de bonitaet es una expresión que se encuentra exclusivamente en estos textos de Kant y que provendría de los escritos de Baumgarten. ${ }^{25}$ En su origen latino, esto es, bonitas, se trata de una sustantivación del carácter de "ser bueno". Como traducción al español podría utilizarse la palabra "bondad", tomando, sin embargo, ciertas precauciones indispensables. Esto significaría, por una parte, que el concepto debe entenderse dejando de lado el sentido coloquial que liga la palabra "bondad" con ideas como la de ser "bondadoso" en cuanto ser "buena persona" o tener un "buen corazón". Es así como la bonitaet debe ser entendida exclusivamente como una sustantivación del carácter general de ser bueno; sólo de esta manera se entiende que, para Kant, la "bondad", i.e. la bonitaet, no sea una atribución de las personas, sino de las acciones.

Por otra parte, el término debe desprenderse de su exclusivo sentido moral: algo es bueno o posee bonitaet en muchos otros sentidos además del moral. En este marco cabe la distinción kantiana entre "bonitaet problemática", "bonitaet pragmática" y "bonitaet moral o absoluta". Una acción es buena en sentido "problemático" cuando ella es un buen medio para alcanzar una finalidad. Cuando ese objetivo es la felicidad, la acción será "pragmáticamente buena". Ahora bien, cuando la acción es buena en sí misma, esto es, que su bonitaet no depende de un fin ulterior, Kant habla de "bonitaet moral o absoluta". Este último tipo de bonitaet no tiene relación

\footnotetext{
${ }^{22}$ La distinción entre diiudicatio y executio aparece por todas partes en las Lecciones y en el Legado manuscrito. Cabe hacer notar, sin embargo, que son conceptos que en los escritos publicados por Kant simplemente no se utilizan. Cfr. Collins KGS.XXVII.274 y Mrongovius KGS.XXVII.1422, Gerhardt 46; R.6628 KGS.XIX.117 y R.6988 KGS.XIX.220.

${ }^{23}$ Cfr. R.6972 KGS.XIX.217 y R.6760 KGS.XIX.151.

${ }^{24}$ Powalski KGS.XXVII.197. Cfr. Collins KGS.XXVII.275.

${ }^{25}$ Cfr. Powalski KGS.XXVII.151, 152, 162, 195; Collins KGS.XXVII.255, 257, 264, 268, 299 , 308, 309.
} 
alguna con medios ni fines, ${ }^{26} \mathrm{y}$, por lo mismo, será sólo ella la que dé pie a que surja una acción verdaderamente moral.

En las Lecciones y Manuscritos, sin embargo, no se habla sólo de la bonitaet moral de las acciones, sino también de la "bonitaet moral de la voluntad", es decir, de un "ser moralmente bueno" (Gutsein) del querer. En este punto, por lo tanto, se salta del problema de la diiudicatio al de la executio: el tema ahora es el querer. Una cosa es saber lo que es moralmente bueno y otra muy distinta es querer realmente hacerlo; dicho en forma negativa, no es lo mismo conocer lo que es incorrecto que el querer evitarlo. Es la "bonitaet de la voluntad", según Kant, el "verdadero principio moral", pues, como dice en las Lecciones: "Nada es bueno excepto aquello que tiene una voluntad buena." ${ }^{27}$ En este punto es imposible no hacer el vínculo con las palabras iniciales de la Fundamentación. ${ }^{28}$ La bonitaet moral del hombre es, de acuerdo con la explicación de Kant, un "ser bueno" de la voluntad; es el poseer una "bonitaet de la voluntad". ${ }^{29}$ Dicho "ser bueno" o bonitaet va a depender del "querer mismo": lo que se juzga de una persona no son sus acciones, sino su querer. Tener bonitaet de la voluntad es querer aquellas acciones que contienen bonitaet moral: que son moralmente buenas y sólo porque lo son. En otras palabras, tener bonitaet de la voluntad implica una disposición de la voluntad a querer lo moral.

Esta descripción de la bonitaet de la voluntad coincide con la idea kantiana de una "moralische Gesinunng", esto es, de una "disposición moral del ánimo": "si yo hago algo porque es en sí mismo universalmente bueno, entonces ésa es una Gesinunng moral". ${ }^{30}$ La Gesinunng moral depende de que se quiera una acción simplemente porque ella contiene bonitaet moral, simplemente porque es buena; ${ }^{31}$ luego, la disposición moral de ánimo no es otra cosa que una bonitaet de la voluntad. En la moral, por lo tanto, de lo que se trata es de la disposición moral del ánimo. "La Ética es, entonces — dice Kant—, una filosofía de la disposición del ánimo."32 De esta disposición del ánimo depende la moralidad de la acción. Kant establece sin ambigüedad alguna que, a su juicio, sólo una acción que se hace única y exclusivamente "por" su bonitaet interior es una acción moralmente buena. ${ }^{33} \mathrm{El}$ "por" es una traducción del término alemán "aus" que se utiliza en

\footnotetext{
26 "Todo aquello que universalmente considerado es bueno, es bueno en sí mismo, por lo tanto, sólo la bonitaet moral es un bien en sí mismo" (R.6700 KGS.XIX.135).

27 Powalski KGS.XXVII.130.

${ }^{28}$ Cfr. Fundamentación KGS.IV.393-395.

${ }^{29}$ Cfr. Mrongovius KGS.XXVII.1410, 1415; Collins KGS.XXVII.266; Powalski KGS.XXVII. 130.

${ }^{30}$ Collins KGS.XXVII.262; Mrongovius KGS.XXVII.1446.

${ }^{31}$ R.7050. "Las disposiciones de ánimo se fundan en la bonitaet interna (necesidad) de la acción" (KGS.XIX.235).

${ }^{32}$ Mrongovius KGS.XXVII.147, y Collins KGS.XXVII.299.

${ }^{33}$ Cfr. Collins KGS.XXVII.279, y Mrongovius KGS.XXVII.1448 y 1412.
} 
este contexto para referirse a la razón por la cual debe ser hecha una acción moral, esto es, sólo porque se ha reconocido su bonitaet moral. Solamente "un motivo puro puede producir una acción pura"; 34 sólo una voluntad buena puede querer una acción "por" su bonitaet interna; luego, sólo ella puede actuar moralmente.

Una vez que se comienza a hablar de la voluntad y del querer como fuente única de moralidad de las acciones, el problema central pasa a ser el de la motivación de la acción moral. Kant señala que "todas la acciones son, de acuerdo con la diiudicatio, sin duda necesarias, pero para que esa acción se lleve a cabo es necesario agregar a eso una motivación". ${ }^{35}$ No basta con conocer la moralidad de una acción, sino que hay que quererla. El querer realizar una acción por su bonitaet interna, como se dijo, obedece a la existencia de una voluntad buena, una voluntad que quiere lo que debe querer, simplemente porque es lo bueno. Su motivación o la fuente de su querer es la razón, en tanto que ella establece lo que posee bonitaet. Por supuesto, existen, de acuerdo con Kant, otro tipo de motivaciones; esto es, aquellas que tienen su origen en la sensibilidad. En las Lecciones y Manuscritos se las llama elatere animi o Triebfedern para distinguirlas de los motiva, que tienen su fundamento en la razón. Las motivaciones subjetivas o sensibles, los "resortes", como se les ha llamado en español, no pueden en ninguna circunstancia dar origen a una acción moral. Un acto es moralmente aceptable únicamente cuando se ha llevado a cabo por el impulso de una motivación moral, i.e. de la razón.

Puesto que establece la bonitaet de una acción, la razón tiene que tener también la capacidad de mover a su realización; esto es, utilizando una terminología posterior, la razón debe ser práctica: tiene que determinar la voluntad. El Kant de esta etapa dice más bien que la diiudicatio debe mover a la executio. "El entendimiento - escribe- tiene que tener, por lo tanto, una potestam executoriam también." ${ }^{36}$ De no ser así, la conclusión es que simplemente no es posible una acción verdaderamente moral. La realidad de la moral, debido a su capacidad de producir actos efectivos que puedan ser calificados de morales, depende de que la razón, la diiudicatio, el juicio, sea lo suficientemente fuerte como para mover al querer y, de allí, al actuar. El Kant de esta etapa tenía un interés expreso por vincular su teoría moral con la realidad, con el mundo. Su teoría moral incluía una preocupación evidente por su realidad; ${ }^{37}$ no pretendía conformarse con establecer la manera en que se conocen las leyes de la moralidad, ni buscaba sólo el modo de determinar el "deber ser"; aspiraba también a que las leyes se cumplieran, se encarnaran en acciones concretas. Una

\footnotetext{
${ }^{34}$ Powalski KGS.XXVII.113.

${ }^{35}$ Collins KGS.XXVII.299.

${ }^{36}$ Powalski KGS.XXVII.201.

${ }^{37}$ Cfr. Collins KGS.XXVII.299.
} 
manifestación clara de esta preocupación se encuentra en la manera como la moral de estos textos se funde con la antropología, ${ }^{38}$ ella debería proveer del indispensable conocimiento sobre el ser humano que haga a la moral no sólo posible, sino real.

\section{Fragilitas e infirmitas}

De acuerdo con la opinión de Kant en los textos analizados, el ser humano es, empero, por naturaleza frágil. ${ }^{39}$ Kant utiliza las palabras fragilitas e infirmitas para referirse a esta fragilidad que no es otra cosa más que una debilidad esencial del hombre. Infirmitas apunta en latín a una debilidad corporal que en su sentido figurado designa también una flaqueza de ánimo: pusilanimidad. Por su parte, fragilitas refiere a la idea de ser caduco, efímero, pasajero, quebradizo. Que el hombre sea frágil significa, por tanto, que es quebradizo, pero no en el sentido de que su cuerpo lo sea, sino más bien su voluntad. La voluntad humana es lo que a juicio de Kant es débil por naturaleza. La causa de esta fragilidad de la voluntad se encontraría en la fuerte impronta que sobre ella ejercen los sentimientos, los afectos, las pasiones y las inclinaciones, por cuanto son capaces de influir en ella a tal grado que incluso pueden llegar a determinarla. Los sentimientos, los afectos, las inclinaciones y las pasiones constantemente intentan ser saciados, y en ese esfuerzo incesante buscarán por todos los medios ganar poder sobre la voluntad del hombre, dando origen así, en tanto que consiguen efectivamente su objetivo, a una debilidad esencial del ser humano.

Esta idea se encontraba ya en los escritos precríticos de Kant en los que se señala, sin rodeos, que las pasiones no sólo influyen, sino que determinan directamente la voluntad. ${ }^{40}$ Para el Kant de estos escritos juveniles, el hombre depende de sus pasiones en cuanto que, a su juicio, ellas son, de entre las motivaciones, las más fuertes. "Las pasiones [Triebe] de la naturaleza humana [...] son las fuerzas que mueven [bewegende Kräfte] la voluntad." ${ }^{41} \mathrm{El}$ hombre simplemente actúa de acuerdo con sus pasiones ${ }^{42}$ y la razón no puede hacer nada en contra. "Los esfuerzos de la razón por sobreponerse a ello y por alejar la confusión mediante la luz de la capacidad de juzgar son - señala Kant- como un rayo de sol que es oscurecido e interrumpido por densas nubes." ${ }^{13}$ Esta dependencia será considerada, tanto en los escritos precríticos como en la obra posterior, como un tipo de

\footnotetext{
${ }^{38}$ Cfr. Collins KGS.XXVII.244, y Mrongovius KGS.XXVII.1398.

${ }^{39}$ Mrongovius KGS.XXVII.1442; Collins KGS.XXVII.294; Powalski KGS.XXVII.159, y R.7176 KGS.XIX.264.

${ }^{40}$ Cfr. Teoría general del cielo KGS.I.353, y Nova Dilucidatio KGS.I.400-401.

${ }^{41}$ Enfermedades mentales KGS.II.261.

${ }^{42}$ Cfr. Nova Dilucidatio KGS.I.400-401.

${ }^{43}$ Teoría del cielo KGS.I.356.
} 
enfermedad o locura. ${ }^{44}$ Kant habla al respecto como de una "patología"45 y considera que ello implica un rebajar al hombre ${ }^{46}$ a tal punto que lo sitúa a la misma altura que los animales. ${ }^{47}$

Para Kant, la dependencia del hombre respecto de sus pasiones tiene, en principio, a lo largo de toda su obra, un sentido claramente negativo. No obstante, en las Lecciones surge, además, junto a ese aspecto rechazable, un sesgo positivo. Las pasiones, en cuanto que son manifestaciones naturales, no tienen originalmente un carácter reprobable, ${ }^{48}$ sino que, por el contrario, son básicamente buenas en sí mismas ${ }^{49} \mathrm{y}$, de hecho, pueden ser disfrutadas en tanto permanezcan bajo control. El problema no radica, entonces, en las pasiones ni en los sentimientos ni en los afectos mismos, sino tan sólo en su posible incontrolabilidad. En estos textos, sin embargo, a diferencia de los más juveniles, ellas pueden ser dominadas en la medida en que uno se vuelve "maestro de sí mismo", como dice Kant, en cuanto que se triunfa sobre las propias inclinaciones, aunque no sea posible evitar definitivamente su influjo, cuyo fundamento radica en aquella debilidad originaria de la voluntad humana. A esta debilidad se le llamará en las Lecciones y en los Manuscritos de la misma manera que en la Religión dentro de los límites de la mera razón: "tendencia al mal" [Hang zum Bösen], en cuanto que es una inclinación a dejarse llevar por las pasiones. ${ }^{50} \mathrm{Se}$ trata, sin embargo, sólo de una "tendencia"; existe una propensión, una inclinación, pero ya no una necesidad o una determinación, como lo era en los escritos precríticos. El hombre conserva siempre la alternativa de no hacer el mal. "Los animales — señala— están determinados necesariamente a actuar, pero los hombres no." 51

Debido al solo hecho de que existe esta tendencia, la razón no está en condiciones de mover directa e inmediatamente a la acción. Aquélla no es lo único que influye en la voluntad, no tiene poder exclusivo sobre el querer; esto tendrá enormes consecuencias para la concepción kantiana de la motivación a la acción moral en los textos analizados. Las motivaciones morales [Beweggründe], la razón, constata el autor, no tienen suficiente poder. ${ }^{52}$ "La reflexión no tiene la fuerza de la sensación." ${ }^{53}$ A raíz de que las pasiones, los sentimientos, los afectos, siempre están allí influyendo, el juicio acerca de la bondad moral de una acción no está en condiciones de

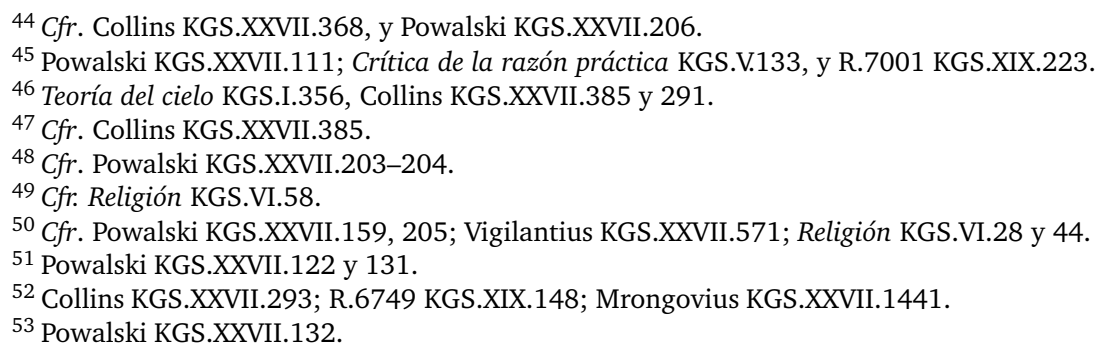


determinar la voluntad por sí sola, pese a ser él quien debería hacerlo. La moral pura sería, entonces, sólo un "ideal" en el sentido de "ficción de perfección"; se encuentra lejos de la realidad. ${ }^{54}$ La conclusión es devastadora: la moral así planteada es sólo una "quimera" que tiene lugar únicamente en la fantasía. "La ética fantástica — señala Kant- es aquella en la que se pretende que las leyes morales, sin intervención de los resortes, sean suficientemente fuertes como para llevar a la acción." 55

La conclusión, en términos de las Lecciones y los Manuscritos, es que la diiudicatio no está en condiciones de mover, por sí sola, a la executio. "Las leyes morales [...] contienen las condiciones objetivas del juicio, pero no las subjetivas de la realización." ${ }^{\text {56 }}$ Es así como a Kant se le presenta como inevitable considerar la incorporación de algún tipo de motivación "subjetiva". Este nuevo motor, sin embargo, ya no podría ser puro ni dar lugar a acciones puramente morales, pero al menos lograría influir en el querer a tal punto que podría efectivamente mover a la acción. La inevitable inclusión de otro resorte por temor a elaborar un pensamiento quimérico lleva al Kant de este periodo a contemplar la posibilidad de la "felicidad" como motivadora de la acción moral. "Las leyes morales — dice- obligan en tanto que están vinculadas con los deseos de felicidad, de lo contrario no tendrían ninguna fuerza para coaccionar." ${ }^{57}$ La moral adquiere realidad mediante la "esperanza de la felicidad" [Hoffnung der Glückseligkeit]. "Es quimérica —dice Kant- toda ética que exija que, en vistas de nuestro comportamiento, no debamos tener esperanza alguna de felicidad." 58

\section{Felicidad y summum bonum}

Ahora bien, lo primero que hay que despejar es lo que Kant está entendiendo aquí por felicidad. Un problema que, por lo demás, aún no ha sido decidido definitivamente es si para Kant existían dos conceptos de Glückseligkeit o sólo uno; si para él junto a una felicidad de tipo físico, basada en la sensibilidad, hay además otra de carácter moral. ${ }^{59}$ Son los escritos mismos de Kant los que, haciendo gala a ratos de una tremenda ambigüedad, dejan abierta la puerta a la duda. Que para Kant la felicidad es algo físico, íntimamente ligado a la sensibilidad, a los sentidos, es indiscutible. No sólo en las Lecciones y en el Legado manuscrito, sino en toda su obra es posible encontrar descripciones que corroboran esta afirmación. La felicidad es de-

\footnotetext{
${ }^{54}$ Cfr. Mrongovius II KGS.XXIX.604, 605.

55 Powalski KGS.XXVII.160. Cfr. Powalski KGS.XXVII.145.

${ }^{56}$ R.7097 KGS.XIX.248. Cfr. Powalski KGS.XXVII.145, y KrV B840/A812.

${ }^{57}$ Powalski KGS.XXVII.167. Cfr. R.7097 KGS.XIX.248.

58 Powalski KGS.XXVII.166.

${ }^{59}$ Para más abundamiento sobre esta discusión, véase José Santos Herceg, "Moral y/o sensible: en torno a la concepción kantiana de felicidad", pp. 39-56.
} 
finida, por un lado, como un "estar contento con el estado o la situación" [Zufriedenheit mit der Zustand], ${ }^{60}$ y, por otro, como la "satisfacción de las inclinaciones" [Befriedigung der Neigungen]. ${ }^{61}$ De lo que se habla aquí es de un sentirse bien en su grado máximo, tanto en relación con la cantidad de alegría como con su duración. En el estado de felicidad, la alegría alcanza su más alto nivel de intensidad y, como si fuera poco, es constante, no reconoce interrupción; es permanente e ilimitada. Las diferentes descripciones de este estado que se pueden encontrar a lo largo de la obra kantiana se complementan. Kant escribe que en él se goza de la alegría ${ }^{62}$ que surge de una conciencia de lo agradable de la vida: ${ }^{63}$ que en él todo se da de acuerdo con las ganas; ${ }^{64}$ que cada deseo es saciado y toda pasión satisfecha.

Que junto a esta concepción de la felicidad como netamente sensible, Kant reconozca la existencia de otra de carácter intelectual o moral, lo propuso por primera vez Klaus Dusing en $1971 .{ }^{65}$ Este autor analiza las Reflexiones sobre filosofía moral de Kant y descubre en ellas un buen número de pasajes en los que parece demostrarse la hipótesis de que para Kant hay otro tipo de felicidad cuya fuente no es ya la simple satisfacción de las pasiones, puesto que su origen es a priori. ${ }^{66}$ Kant habla, en efecto, de una felicidad producida [erzeugt] por la libertad, ${ }^{67}$ en cuanto que la libertad es su fundamento [Grund], ${ }^{68}$ origen [Ursache ${ }^{69}$ y principio [pincipium]. ${ }^{70}$ Esta "verdadera felicidad" proveniente de la libertad, ${ }^{71}$ que no es más que un "efecto de la libertad", ${ }^{72}$ está contrapuesta a la felicidad que es "sólo un efecto casual y dependiente de la naturaleza". ${ }^{73}$ Especialmente interesante es el Manuscrito número 6867, en el que Kant se refiere a la "autocracia de la libertad respecto de la felicidad", a que la "fuente de la felicidad está en posesión de la criatura racional" y a la felicidad como una "creación de la

${ }^{60}$ Cfr. Powalski KGS.XXVII.101. La misma idea se puede encontrar también en la Fundamentación KGS.IV.393, en la Crítica de la razón práctica KGS.V.22 y 124, y en la Metafísica de las costumbres KGS.VI.387.

${ }^{61}$ Cfr. Powalski KGS.XXVII.101, 217, y Mrongovius KGS.XXVII.1424. Véase también en la obra posterior: $K r V$ A 806/B 834 y KpV KGS.V.73 y 147.

${ }^{62}$ Cfr. Powalski KGS.XXVII.101.

${ }^{63}$ Cfr. KpV KGS.V.22.

${ }^{64}$ Cfr. Met. de las cost. KGS.VI.480, y KpV KGS.V.124.

${ }^{65}$ K. Dusing, "Das Problem des höchsten Gutes in Kants praktischer Philosophie", KS 62, pp. 5-42.

${ }^{66}$ R.6911 KGS.XIX.203-204.

${ }^{67}$ Cfr. R.6924 KGS.XIX.177; R.6857 KGS.XIX.181; R.7199 KGS.XIX.272, y R.7204 KGS. XIX.283.

68 R.6844 KGS.XIX.177.

${ }^{69}$ R.7199 KGS.XIX.272-274, y R.7200 KGS.XIX.274.

${ }^{70}$ R.6867 KGS.XIX.186, y R.7209 KGS.XIX.285-286.

${ }^{71}$ R.7199 KGS.XIX.272.

72 R.7205 KGS.XIX.284.

${ }^{73}$ R.6907 KGS.XIX.202. Cfr. R.6867 KGS.XIX.186; R.6913 KGS.XIX.204; R.7202 KGS.XIX. 276-282, y R.7260 KGS.XIX.296-297. 
propia voluntad buena". ${ }^{74}$ De acuerdo con este texto, el virtuoso lleva la felicidad en sí mismo, en tanto que la conciencia de ser virtuoso ya es un tipo de felicidad. ${ }^{75}$

Esta misma idea reaparecerá en la obra posterior de Kant con una estructura muy similar, esto es, en La religión dentro de los límites de la mera razón ${ }^{76}$ y en la Metafísica de las costumbres. ${ }^{77}$ Lo interesante es, sin embargo, que en estos textos maduros Kant se apresurará a establecer expresamente que algo así como una "felicidad moral" es una "quimera contradictoria" [widersprechendes Unding], ${ }^{78}$ porque de hecho "contiene una contradicción en sí misma". ${ }^{79}$ Para el Kant de la obra publicada, la felicidad es esencialmente física, sensible, y si se refiere a lo que "algunos autores" han llamado felicidad moral, es sólo con la intención de desacreditar el concepto como insostenible. Esta actitud, sin embargo, no hace más que hacerse eco de las palabras que se leen ya en el Legado manuscrito: "acerca de una felicidad moral — dice allí — no entendemos nada" ${ }^{80}$ Ya entonces pensaba que una felicidad que no tuviera nada que ver con la sensibilidad no podía ser considerada verdadera felicidad. Las alusiones a una felicidad intelectual en el Legado manuscrito, sobre las que Düssing basa su hipótesis, deben ser entendidas correctamente. En ninguna parte se habla, de hecho, de la "felicidad intelectual", sino solamente de "lo intelectual de la felicidad", entendiendo con ello que se trata de una faceta, de una parte de la felicidad total. ${ }^{81}$ Lo moral puede ser una cara de la felicidad que tal vez podría llamarse intelectual; la otra es, no obstante, siempre sensible, física, estética, empírica. La felicidad en su mayor expresión no es, para Kant, más que un estado en que las pasiones son saciadas.

Es de acuerdo con esta idea de felicidad que Kant acepta y sostiene que ella es el fin natural e ineludible del hombre. ${ }^{82}$ La manera en que distingue, ya desde los escritos precríticos, ${ }^{83}$ entre los diferentes tipos de imperativos -dejando con ello expresamente establecido que el imperativo de astucia, sagacidad o prudencia [Klugheit] es sólo un "consejo" y jamás podría tener carácter de universal y necesario- hace manifiesto, sin embargo, que

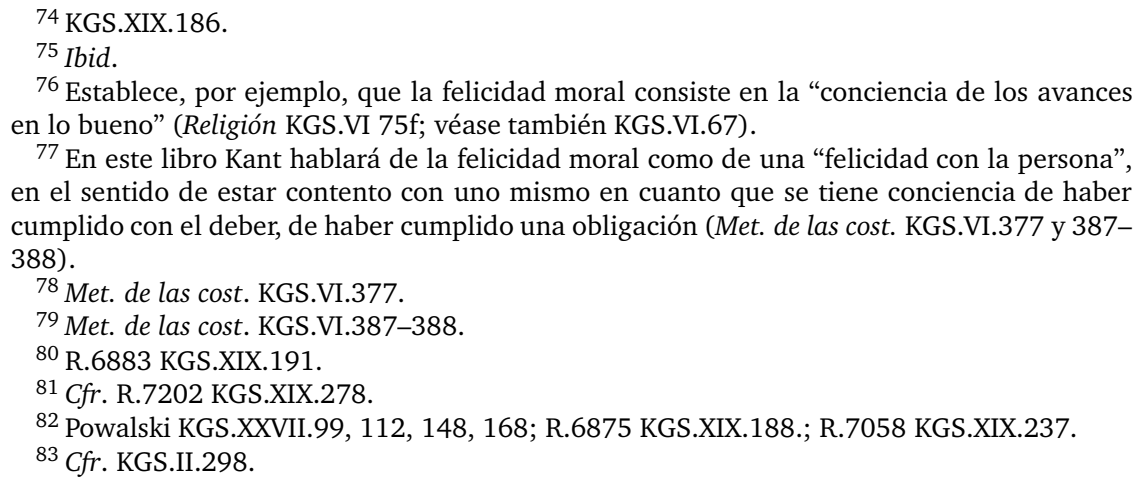

${ }^{76}$ Establece, por ejemplo, que la felicidad moral consiste en la "conciencia de los avances en lo bueno" (Religión KGS.VI 75f; véase también KGS.VI.67).

${ }^{77}$ En este libro Kant hablará de la felicidad moral como de una "felicidad con la persona", en el sentido de estar contento con uno mismo en cuanto que se tiene conciencia de haber cumplido con el deber, de haber cumplido una obligación (Met. de las cost. KGS.VI.377 y $387-$ 388).

${ }^{78}$ Met. de las cost. KGS.VI.377.

${ }^{79}$ Met. de las cost. KGS.VI.387-388.

${ }^{80}$ R.6883 KGS.XIX.191.

${ }^{81}$ Cfr. R.7202 KGS.XIX.278.

82 Powalski KGS.XXVII.99, 112, 148, 168; R.6875 KGS.XIX.188.; R.7058 KGS.XIX.237.

${ }^{83}$ Cfr. KGS.II.298. 
Kant siempre conoció los riesgos y problemas del eudemonismo, ${ }^{84}$ y que su intención nunca pudo haber sido que su teoría moral entrara en esa categoría. Pese a ello, Hermann Schmitz ha sostenido sin ambigüedad alguna que la teoría de la acción de Kant, entre 1764 y 1784, no es más que un eudemonismo. ${ }^{85}$ Esta opinión, por lo demás, no es nueva, pues ya en 1895 Osias Thon sostenía lo mismo, ${ }^{86}$ y Schopenhauer afirmaba que Kant habría logrado erradicar el eudemonismo de su ética más en apariencia que en realidad. ${ }^{87}$ Por último, Rodríguez Aramayo ${ }^{88}$ y Ada Lamacchia ${ }^{89}$ sostienen una interpretación semejante.

El eudemonismo de Kant en este periodo, por lo tanto, parece ser un tema abierto, no decidido del todo; un problema del que, por lo demás, el autor era perfectamente consciente, a juzgar por la gran presencia que tienen en su textos tanto la crítica a esta posición, como el tratamiento del asunto del bien supremo. En efecto, mediante la incorporación de este último tema, Kant intentará que la felicidad concurra como apoyo en la motivación de la acción moral, sin caer con ello en un eudemonismo flagrante. En el concepto de summum bonum, Kant pretenderá sintetizar las dos mayores plenitudes: la felicidad y la virtud. En el marco de la discusión acerca de la manera en que debieran articularse estas dos plenitudes, el autor reproduce su dilema. Por un lado está la alternativa de una "moral ideal", en la que se sostiene, como él ha hecho, que sólo una acción que se hace por un motivo puro es moral, y por el otro está la posibilidad de una "moral real", en la que la finalidad de todo acto sea la felicidad. En otras palabras, la alternativa se mueve entre Zenón y Epicuro, de acuerdo con la forma en que el mismo Kant expone sus respectivas teorías. La primera posibilidad contiene toda la pureza necesaria, pero carece absolutamente de realidad; a la segunda le falta pureza y valor moral, pero tiene efectos en la práctica. ${ }^{90}$

El reto para Kant era dar con una "tercera" alternativa en la que tanto la pureza como la realidad estuvieran presentes. Tenía que encontrar una manera de coordinar ambas finalidades; con ello tendría en su mano la más pura de las motivaciones posibles para la acción moral y, simultáneamente,

84 "Existe una meta que todos los hombres tienen, ésta es la felicidad; sin embargo, está poco claro qué es para cada uno la felicidad" (Powalski KGS.XXVII.99). Cfr. 6935 KGS.XIX.209-210; Mrongovius KGS.XXVII.1400, 1405.

${ }^{85}$ Cfr. Hermann Schmitz, Was wollte Kant?, pp. 81 y 85.

${ }^{86}$ Cfr. Osias Thon, Die Grundprinzipien der Kantischen Moralphilosophie in ihrer Entwicklung, pp. 23-25.

${ }^{87}$ Cfr. A. Schopenahuer, "Preisschrift über die Grundlage der Moral", p. 643.

88 Rodríguez Aramayo, Roberto; "La presencia de la Crítica de la razón práctica", p. 152.

${ }^{89}$ Ada Lamacchia, "Ethik und Religion in den Vorlesungen von Kant (1762-64; 1775-80; 1783-84)", pp. 513-517.

90 "Epicuro tomó los fundamentos subjetivos de la executio que nos mueve a la acción como fundamentos objetivos de la diiudicatio. Zenón, todo lo contrario" (R.6619 KGS.XIX.112). 
la más efectiva. Sin entrar aquí en un análisis detallado de las diferentes alternativas entre las que se mueve Kant para intentar su propósito de articular virtud y felicidad, ${ }^{91}$ diremos simplemente que el autor desecha todo tipo de vinculación lógica (identidad) ${ }^{92}$ y se inclina por una de carácter "sintético", en cuanto que ambos términos quedan unidos por un vínculo de "causalidad". No obstante, es importante señalar que la "causalidad" en principio tampoco es una buena solución para Kant. El que la felicidad sea causa, es decir, que sea lo que mueva a la virtud, es absolutamente imposible, dice, y el que la virtud sea la causa de la felicidad también es falso. Efectivamente, la virtud no puede ser considerada "causa" de la felicidad, pues, de hecho, constata Kant, generalmente ocurre todo lo contrario. ${ }^{93}$ Esta segunda alternativa, sin embargo, no sería del todo falsa, sino, usando una expresión tardía, sería sólo "condicionadamente falsa" [bedingterweise falsch]. ${ }^{94}$

Ésta será la posibilidad que explore Kant. Para ello tendrá que mostrar que es posible que la virtud sea la "causa" de la felicidad, en cuanto que es su "fuente"; que al ser virtuoso resulta también la felicidad y que no por ello se transforma la virtud en un medio para alcanzarla. Tendrá que poder hacer comprensible el hecho de que la virtud conduzca a la felicidad, sin que ello implique que la felicidad sea el objetivo que se pretende alcanzar mediante ella. El elemento central aquí será la concepción de la virtud en cuanto "Glückswürdigkeit": "iSer virtuoso! significa: compórtate de tal forma que te hagas digno de la felicidad". ${ }^{95}$ La dignidad de ser feliz debe ser entendida en el sentido del "mérito": en la medida en que se es virtuoso uno se hace merecedor de la felicidad. La virtud, sin embargo, sólo nos hace "dignos" de la felicidad, lo que no significa aún que se la alcanzará necesariamente. La felicidad se encuentra, por así decirlo, al final del camino, "como si" fuera una meta que por nada del mundo debe ser entendida como tal. Kant insiste en que se trata sólo de una "consecuencia posible" [mögliche Folge]. En las Lecciones se utiliza una serie de términos que buscan aprehender esta delicada situación: es así como se habla de consequentia, ${ }^{96}$ correlatum $^{97}$ o consectaria.$^{98}$ El objetivo es establecer una vinculación entre la virtud y la felicidad que no implique que la primera sea un medio para alcanzar la segunda. Eso se logra debido a que no se

\footnotetext{
${ }^{91}$ Véase un resumen en $c f r . K p V$. KGS.V.111.

92 Cfr. Mrongovius KGS.XXVII.1402.

93 Mrongovius II. KGS.XXIX.623.

${ }^{94} \mathrm{KpV}$ V.114.

95 Mrongovius II KGS.XXIX.601-602. Cfr. KrV B 613, 834, 838, 840/A 585, 806, 810, 812. Cfr. también KpV KGS.V.110; KU KGS.V.450 y KGS.V.452; Religion KGS.VII.65; Met. de las cost. KGS.VI.457 y VI.482. Lecciones KGS.XXVII.166, 167, 209.

96 Powalski KGS.XXVII.148.

97 Powalski KGS.XXVII.167.

98 Powalski KGS.XXVII.151.
} 
trata de un nexo "necesario", sino sólo "posible". La virtud no es "causa", sino "condición" necesaria de una felicidad que no es "efecto" ni "objetivo", sino sólo "consecuencia posible".

De esta forma la felicidad sería algo que se puede tener la "esperanza" de alcanzar en tanto se cumpla con los requisitos, pero que no se obtiene necesariamente, al modo de una "ganancia" o una "remuneración debida". "El hombre — dice Kant- puede tener la esperanza de ser feliz; esto, sin embargo, no puede moverlo, sino sólo aliviarlo. Aquel que vive moralmente puede tener la esperanza, por ello, de que será recompensado." ${ }^{99} \mathrm{La}$ felicidad es lo que Kant llama praemia remuneratia. ${ }^{100}$ No puede exigírsela pese a haber sido alcanzada la necesaria dignidad, el requerido "mérito". La virtud es la condición, pero la felicidad es sólo una consecuencia posible. A quien es digno de la felicidad solamente le queda tener esperanza (hoffen, erwarten, wünschen). De esta forma se busca lograr que la felicidad no sea lo que motive a la acción. ${ }^{101}$ La esperanza de felicidad es un "motiva subsidiaria", esto es, un motivo que no es el decisivo, ${ }^{102}$ porque, de hecho, es algo incierto.

\section{Dios: apoyo y garante}

Dos problemas se le presentan a Kant en este punto. Por un lado, está el asunto de la efectiva posibilidad de llegar a ser virtuoso, esto es, alcanzar la dignidad necesaria. En efecto, dependiendo de la concepción de virtud de la que se esté hablando, existe la posibilidad de que la "condición" nunca se logre cumplir. Por otro lado, aparece el problema del fundamento de la esperanza: ¿sobre qué fundo mi esperanza de que siendo virtuoso podré, tal vez, ser feliz? Está bien que la felicidad no pueda ser la meta, pero si ha de influir de alguna manera en la voluntad, la esperanza de alcanzarla debe tener algún asidero. Dos problemas, una sola solución: Dios.

Respecto del primero de estos temas, habrá que comenzar por delimitar el tipo de virtud del que se está hablando. La distinción entre una virtud simple y una virtud perfecta o pura está presente en todos los escritos de Kant. Por supuesto que la "pureza" y la "perfección" de la virtud dependen de que se tenga una voluntad absolutamente buena, es decir, perfecta; ${ }^{103}$ esto es lo que se llamará "santidad". ${ }^{104}$ En el plano de la concepción del bien supremo, la distinción es sumamente relevante, pues si la dignidad

\footnotetext{
${ }^{99}$ Collins KGS.XXVII.284.

100 Collins KGS.XXVII.284; Mrongovius KGS.XXVII.1434.

${ }^{101}$ Cfr. Mrongovius II XXIX.624.

${ }^{102}$ Cfr. Collins KGS.XXVII.303.

${ }^{103}$ Cfr. Mrongovius II KGS.XXIX.599, Powaslki KGS.XXVII.130, R.7216 KGS.XIX.287 y Fundamentación KGS.IV.393.

${ }^{104}$ Cfr. KpV KGS.V.84; Met. de las cost. KGS.VI.405 y 409, Mrongovius KGS.XXVII.1448.
} 
requerida para aspirar a la felicidad es una virtud simple, entonces no hay problema: todo el mundo puede alcanzarla y el grado de mérito obtenido habrá de ser proporcional al nivel de virtud que se posea. Pero si la condición es una virtud perfecta, está claro que el hombre no puede alcanzar este grado de perfección moral y, por lo tanto, nunca se hará del todo digno de la felicidad: "todos tienden a acercarse a la virtud — dice Kant- [...], pero ninguno alcanza el grado máximo". ${ }^{105}$

Para superar esta dificultad, Kant utiliza en las Lecciones y en los Manuscritos la idea de la "Gottes Beihilfe" o "Beistand Gottes", ${ }^{06}$ que puede traducirse como "ayuda, asistencia divina" o "apoyo de Dios". Con ello busca mostrar que, si bien el hombre no está en condiciones de alcanzar con sus propias fuerzas la virtud perfecta, sí le es posible llegar a ella con la ayuda divina. Es Dios quien complementa [ersetzt] las falencias del ser humano, haciendo posible que llegue a ser plenamente virtuoso. No se trata, sin embargo, de una ayuda gratuita; para poder recibirla, el ser humano debe hacer primero todo lo que esté en sus manos para ser virtuoso. ${ }^{107}$ También aquí hay que hacerse digno, es decir, merecedor de ese regalo, ${ }^{108}$ y la obtención de esta ayuda, una vez más, es sólo objeto de una esperanza que depende de la "bondad" divina; ${ }^{109}$ en ningún caso es exigible. Si hacemos todo lo que está en nuestro poder para llegar a hacernos dignos del apoyo divino, podemos "tener la esperanza" de que Dios tiene los medios para superar nuestra "imperfección". 110

Luego de alcanzar, con la indispensable ayuda de Dios, la virtud, esto es, la dignidad necesaria, el virtuoso queda nuevamente abandonado solo a su esperanza. En este punto abordamos el segundo problema a que nos referíamos antes, es decir, al problema de la fundamentación de la esperanza de ser feliz. La felicidad es, como quedó aclarado, sólo una consecuencia posible de una virtud que tiene el carácter de "condición necesaria", pero no suficiente, de la felicidad. Dios será en esta etapa el "garante" de que la felicidad pueda ser alcanzada si existe la correspondiente dignidad. ${ }^{111}$ Él garantiza que aquel que ha alcanzado la virtud pueda esperar fundadamente llegar a ser también feliz, en tanto que es Él quien premia. ${ }^{112}$

105 Collins KGS.XXVII.463 y Mrongovius KGS.XXVII.1576. "La virtud es una idea y nadie puede poseer verdadera virtud" (Collins KGS.XXVII.463). Cfr. Mrongovius KGS.XXVII.1576, y KpV KGS.V.122.

106 Mrongovius KGS.XXVII.1463-1464, y Collins KGS.XXVII.320-321.

${ }^{107}$ Cfr. Collins KGS.XXVII.331; Mrongovius KGS.XXVII.1463.

108 "Sin embargo, podemos esperar una ayuda bondadosa en vistas a mejorar nuestra voluntad si es que hacemos todo lo que esté en nuestras manos y nos comportamos ante la santa Ley [... ] con humildad" (R.7093 KGS.XIX.247).

${ }^{109}$ Mrongovius KGS.XXVII.1464.

${ }^{110}$ Mrongovius KGS.XXVII.1461, 1487; Collins KGS.XXVII.317.

${ }^{111}$ Es interesante hacer ver que la reflexión moral presente en la Crítica de la razón pura pertenece también a esta etapa del desarrollo del pensamiento de Kant. Cfr. KrV B 838/A 810. ${ }^{112}$ Cfr. Powalski KGS.XXVII.168. 
"Reconocemos así que aquel que se ha comportado de tal manera que se ha hecho digno de la felicidad, también puede tener la esperanza de alcanzarla, porque existe un ser que puede hacer feliz."113

Que un Dios sea quien premie con la felicidad no puede significar, sin embargo, que sea él quien decida arbitrariamente a quién se la otorga. Para Kant, debe tratarse, ante todo, de un Dios justo. ${ }^{114}$ La justicia divina no admite excepciones; ${ }^{115}$ no es un juez "bueno", sino "justo". ${ }^{116}$ Desde este punto de vista, no hay compasión. Como juez, Dios será "temido", pues cada uno recibirá lo que merece. ${ }^{117}$ Esta justicia divina es, por lo tanto, una justicia distributiva. Dios, como juez justo, garantiza que cada uno reciba aquello de lo que se ha hecho digno. Los virtuosos pueden, entonces, tener esperanzas fundadas de que obtendrán la felicidad.

De esta forma Dios se vuelve a tal punto necesario que Kant llega a decir que "si nos faltara un ser que realizara todo esto, nuestra moral no tendría realidad alguna, sino que sería una mera y simple idea". ${ }^{118}$ Tanto la esperanza de la felicidad que depende de la virtud (dignidad), como la esperanza de alcanzar esa virtud, dependen al final de la suposición [Annahme] de la existencia de un Dios. De un Dios que, en tanto que justo, otorgue la felicidad a aquellos que se han hecho dignos de ella, y, en tanto que bueno, ayude a ser dignos (virtuosos) a aquellos que hacen todo lo posible por llegar a serlo.

La religión adquiere así un papel fundamental. Será el propio Kant quien, al analizar los errores de los antiguos en su afán por lograr un concepto coherente de bien supremo, diga expresamente, tanto en las Lecciones como en el Legado manuscrito, que tales errores se deben a que pretenden dejar a la religión fuera. Dios es, en estos textos, quien hace posible tanto la condición (virtud) como la consecuencia o premio (felicidad). No puede caber duda acerca de su papel fundamental para la concepción del bien supremo y, por lo tanto, para la moral kantiana de esta época. Él lo dice textualmente: "La moral tiene que estar unida con la religión". ${ }^{119}$ De acuerdo con sus escritos, la religión da, a través del concepto de bien supremo, "belleza", "vehemencia" y "realidad" a la moral. ${ }^{120}$ Por lo tanto, la moral

113 Collins KGS.XXVII.308, y Powalski KGS.XXVII.179.

114 "La justicia divina es la repartición precisa de los castigos y los premios de acuerdo con el bien o la buena acción de los hombres" (Collins KGS.XXVII.331).

115 Cfr. Collins KGS.XXVII.331.

116 R.7174. "Un juez bueno es una contradictio in adiecto" (KGS.XIX.264).

117 "Nuestro comportamiento respecto de Dios es de tres tipos: podemos honrarlo, temerle y amarlo. Honramos a Dios en cuanto santo legislador, lo amamos en cuanto buen gobernante, y le tememos en cuanto juez justo" (Collins KGS.XXVII.322).

118 Powalski KGS.XXVII.137.

119 "La eticidad tiene que estar unida con la religión" (Collins KGS.XXVII.307).

${ }^{120}$ Mrongovius KGS.XXVII.1453, y Collins KGS.XXVII.307. 
sin religión está "incompleta", ${ }^{121}$ porque sólo la religión tiene el poder de otorgarle realidad, ${ }^{122}$ en cuanto que de ella depende la motivación de la acción moral. La religión hace que la moral sea "práctica", esto es, que mueva a la acción. ${ }^{123}$

Esto no significa, sin embargo, que aquí se esté en presencia de una "moral teonómica" o "teológica". Con este nombre se suele aludir a una concepción de la moral en que Dios es tanto el que da la ley como el que juzga sobre su cumplimiento. En este tipo de teorías, la divinidad es a un tiempo legislador y juez. El que Kant postule en las Lecciones y las Reflexiones una teoría de este tipo es insostenible, pues, pese a que, como mostramos, Dios es juez, en ningún caso es quien crea la ley moral. Ella emana, según Kant, única y exclusivamente de la razón. Cabe preguntarse, entonces, cómo es posible que Dios juzgue de acuerdo con una ley que no ha emanado de su voluntad. La respuesta kantiana pasa por la idea de que existe una "Übereinstimung" (coincidencia) entre la ley divina y la ley moral. ${ }^{124}$ "Las Leyes de la voluntad divina — dice- son las Leyes morales". ${ }^{125}$ Según esta idea, quien actúa moralmente también lo hace de acuerdo con las leyes de Dios, pues "las leyes morales [...] pueden ser mandamientos de la voluntad divina, pero ellas no emanan de los mandamientos. Dios lo ha mandado, porque es una ley moral, y su voluntad coincide con las leyes morales." ${ }^{26}$ Dios no es quien promulga la ley moral y, sin embargo, en tanto que esta ley está de acuerdo con su intención, puede ser vista como una ley suya.

Para aclarar esta situación, Kant utiliza la distinción entre "Gesetzgeber" (legislador) y "Urheber" (creador) de la ley moral. Dios es el legislador de la ley moral, pero no puede ser visto, en ninguna circunstancia, como su creador, ${ }^{127}$ pues una ley necesaria y universal, como lo es la ley moral, no puede tener un creador determinado. ${ }^{128}$ "De las leyes morales -dice Kant- nadie es el creador, ni siquiera la divinidad misma, porque ellas no surgen del arbitrio, sino que son prácticamente necesarias." 129 La ley moral no emana de la voluntad divina; ${ }^{130}$ Dios se limita a señalar, por una parte, que actuar de acuerdo con ella es la condición para tener una voluntad

${ }^{121}$ Powalski KGS.XXVII.164.

${ }^{122}$ Cfr. Mrongovius KGS.XXVII.1453.

${ }^{123}$ Powalski KGS.XXVII.137.

124 “[L]a moralidad subjetiva de Dios coincide, por lo tanto, con la moralidad objetiva, y cuando actuamos de acuerdo con la moralidad objetiva, entonces actuamos también de acuerdo con la voluntad divina" (Collins KGS.XXVII.263).

125 Powalski KGS.XXVII.135.

${ }^{126}$ Collins KGS.XXVII. 277.

${ }^{127}$ Cfr. Powalski KGS.XXVII.146-147. Véase también Powalski KGS.XXVII.169, y Collins KGS.XXVII.283.

128 Powalski KGS.XXVII.145.

129 Collins KGS.XXVII.283, y Mrongovius KGS.XXVII.1433.

${ }^{130}$ Powalski KGS.XXVII.136. 
buena, y, por otra, que estas leyes están de acuerdo con su voluntad. ${ }^{131}$ Dios no es el creador de la ley moral, pero permanece vinculado a ella como legislador. ${ }^{132}$ Así es como se comprende tanto la coincidencia de ambas normativas como el hecho de que Dios esté habilitado para ser el juez que determine su cumplimiento.

\section{Conclusión}

Con la ayuda de los textos de las Lecciones y el Legado manuscrito se ha pretendido destacar algunos elementos especialmente significativos que nos permiten intentar una suerte de reconstrucción arqueológica inicial de la concepción moral de Kant durante este periodo de aparente silencio (1770-1781). Es así como, sin desconocer las dificultades propias de estas fuentes, se ha llamado la atención sobre los conceptos de diiudicatio, executio y bonitaet como constitutivos de una suerte de teoría de la acción moral. Asimismo, se ha visto la manera como pugnan en la reflexión kantiana de esta etapa una concepción pura de la moral con una que pretende ser real, haciéndose cargo de la debilidad propia de la voluntad humana. Finalmente, sobre la base de la concepción kantiana del bien supremo se ha apuntado al papel gravitante que tienen Dios y la religión para la concepción moral de Kant durante esta época. Estos elementos son sólo algunos de los materiales que sobre este tema se pueden encontrar en las Lecciones y los Manuscritos, en el entendido de que no es tan evidente que en lo relativo a estos escritos y a este periodo se pueda efectivamente hablar de la moral kantiana en singular, ni de la concepción de la moral de Kant como si se tratara de un sistema completo y articulado. Lo que hay en estos textos son más bien problemas, dudas y ensayos de posibles soluciones, rectificaciones, bocetos, esquemas. Como se podrá haber notado, algunos elementos sobreviven, y se pueden observar más o menos nítidamente en los escritos morales publicados por Kant. Otros quedaron enterrados y olvidados bajo resmas de papeles. Sin embargo, si es verdad lo que el mismo Kant ha señalado, en el sentido de que "no es posible aprender filosofía sino a filosofar", ${ }^{133}$ la mejor escuela es, sin duda, asistir al "taller del espíritu" de Kant, como llamó Adickes a estos textos; echar un vistazo a aquel lugar donde germinaron y se desarrollaron sus pensamientos, para aprender allí a filosofar.

${ }^{131}$ R.7089. "Dios no hace las leyes morales o la obligación (Él las da), sino que sólo dice que ellas son las condiciones de una voluntad buena" (KGS.XIX.246).

${ }^{132}$ Cfr. Powalski KGS.XXVII.145-146, y Mrongovius KGS.XXVII.1433.

${ }^{133}$ Cfr. KrV A 838/B 866. 


\section{BIBLIOGRAFÍA}

Aniz Iriarte, C., "Proceso evolutivo hacia la Crítica de la razón práctica", Estudios Filosóficos (Valladolid), vol. 37, 1988, pp. 65-79.

Beiser, F.C., "Kant's Intellectual Development 1746-1781", en The Cambridge Companion to Kant, ed. Paul Guyer, Cambridge, 1992, pp. 26-61.

Foerster, Fr.W., Der Entwicklungsgang der Kantischen Ethik bis zur Kritik der reinen Vernunft, Mayer and Müller, Berlín, 1893.

Forschner, M., "Moralität und Glückseligkeit in Kants Reflexionen", Zeitschrift für philosophische Forschung, vol. 42, 1988, pp. 397-416.

_ - "Das Ideal des moralischen Glaubens. Religionphilosophie in Kants Reflexionen", en Friedo Riken y François Marty (comps.), Kant über Religion, Kohlhammer, Stuttgart, 1992, pp. 83-99.

Heimsoeth, Henrich y G. Tonelli (comps.), Studien zu Kants philosophischer Entwicklung, G. Olms, Hildesheim, 1967.

Hinske, N., Kants Weg zur transzendentalephilosophie. Der dreißigjährige Kant, Kohlhammer, Berlín, 1970.

Kant, Immanuel, Crítica de la razón pura, trad. Pedro Rivas, Alfaguara, Madrid, 1993.

—_ Eine Vorlesung über Ethik, Fischer Taschenbuch Verlag, Fráncfort, 1991.

—_ Lecciones de ética, trad. Roberto Rodríguez Aramayo y Concha Roldán Panadero, Crítica, Barcelona, 1988.

—_, Reflexiones sobre filosofía moral, trad. José Santos Herceg, Sígueme, Salamanca, 2004.

—, Sämmtliche Werke, ed. Rosenkranz y Schubert, Leipzig, 1838.

—_ Sämmtliche Werke in chronologischer Reihenfolge, ed. Hartestein, Leipzig, 1867.

Küenburg, M., Ethische Grundfragen in der jüngst veroffentlichen Ethikvorlesung Kants, Kohlhammer, Innsbruck, 1925.

Lamacchia, A., "Ethik und Religion in den Vorlesungen von Kant (1762-64; 177580; 1783-84)", Akten des XIV Internationalen Kongresses für Philosophie (Viena, 2-9 de septiembre de 1968), Herder, Viena, 1970, tomo V, pp. 513-517.

Lee, M.-H., Das Problem des Moralischen Gefühls in der Entwicklung der kantischen Ethik (Bonn, 1987), Taipei, 1994.

Lehmann, G., "Einleitung zu Kants Vorlesungen über Moralphilosophie", Kants Gesammelte Schriften, vol. XXVII, 1036.

__ Beiträge zur Geschichte und Interpretation der Philosophie Kants, de Gruyter, Berlín, 1969.

— - Kants Tugenden, Neue Beiträge zur Geschichte und Interpretation der Philosophie Kants, de Gruyter, Berlín, 1980.

__ , "Kants Bemerkungen im Handexemplar der Kritik der praktischen Vernunft", Kant-Studien, vol. 72, 1981, pp. 132-139.

Menzer, P., Der Entwicklungsgang der Kantischen Ethik bis zum Erscheinen der Grundlegung der Metaphysik der Sitten, Berlín, 1897.

_- "Der Entwicklungsgang der Kantischen Ethik in den Jahren 1760 bis 1785", Kant-Studien, vol. 2, 1899, pp. 290-322, y Kant-Studien, vol. 3, 1899, pp. 41104. 
Rodríguez Aramayo, R., "La presencia de la Crítica de la razón práctica en las 'Lecciones de Ética' de Kant”, Ágora, papeles de filosofía (Santiago de Compostela), 1988, pp. 145-158.

_ _ "La cara oculta del formalismo ético", en Immanuel Kant, Lecciones de Ética, Crítica, Barcelona, 1988, pp. 7-34.

Sala, G.B., "Die Veröffentlichung der Vorlesungsnachschriften Kants in der Akademische-Ausgabe", Theologie und Philosophie (Friburgo), vol. 57, 1982, pp. $202-$ 224.

Santos Herceg, José, "Moral y/o sensible: en torno a la concepción kantiana de felicidad", Teorema, vol. 23, no. 1-3, 2004, pp. 39-56.

_- "Reivindicación de las Lecciones y Reflexiones como fuentes de la filosofía moral de Kant", Veritas. Revista de filosofía de la PUCRs, Porto Alegre, vol. 46, no. 4, 2001, pp. 555-570.

Schilpp, P.A., Kant's Pre-Critical Ethics, 2a. ed., Northwestern University Press, Evanston, 1970 (Chicago, 1938). [Hay versión en castellano: La ética precrítica de Kant, 2a. ed., trad. Jerónimo Muñoz y Elsa Cecilia Frost, Instituto de Investigaciones Filosóficas-UNAM, 1997.]

Schmidt, K., Beiträge zur Entwicklung der Kantischen Ethik, Marburgo, 1900.

Schmitz, H., Was wollte Kant?, Bouvier, Bonn, 1989.

Schmucker, J., Die Entwicklung der Lehre Kants von den Prinzipien der Moralität bis zur "Kritik der reinen Vernunft", Diss., Munich, 1948.

- Die Ursprünge der Ethik Kants in seinen vorkritischen Schriften und Reflexionen, Hain, Meisenheim am Glan, 1961.

Schopenhauer, A., "Preisschrift über die Grundlage der Moral", Arthur Schopenhauer Kleinere Schriften, Sämtliche Werke, tomo III, Suhrkamp, Stuttgart/Fráncfort del Meno, 1962.

Thon, O., Die Grundprinzipien der Kantischen Moralphilosophie in ihrer Entwicklung, Berlín, 1895.

Vázquez Lobeiras, M.J., "Nueva propuesta para una lectura genética de la obra de Kant", Daimon, no. 8, 1994, pp. 189-194.

Ward, K., The Development of Kant's View of Ethiks, Blackwell, Oxford, 1972.

Werkmeister, W.H., Kant's Silent Decade. A Decade of Philosophical Development, University Presses of Florida, Tallahassee, 1979.

- The Architectonic and Development of his Philosophy, Open Court, Londres, 1980.

Recibido el 17 de diciembre de 2004; aceptado el 29 de marzo de 2005. 